\title{
Reduction of multiple scattering of high-energy positively charged particles during channeling in single crystals
}

\author{
W. Scandale ${ }^{1}$, L. S. Esposito ${ }^{1}$, M. Garattini ${ }^{1,9}$, R. Rossi ${ }^{1}$, V. Zhovkovska ${ }^{1}$, A. Natochii ${ }^{2}$, F. Addesa ${ }^{3}$, F. Iacoangeli ${ }^{3}$, \\ F. Galluccio ${ }^{4}$, F. Murtas ${ }^{5}$, A. G. Afonin ${ }^{6}$, Yu. A. Chesnokov ${ }^{6}$, A. A. Durum ${ }^{6}$, V. A. Maisheev ${ }^{6, a}$, Yu. E. Sandomirskiy ${ }^{6}$, \\ A. A. Yanovich ${ }^{6}$, G. I. Smirnov ${ }^{1,7}$, Yu. A. Gavrikov ${ }^{8}$, Yu. M. Ivanov ${ }^{8}$, M. A. Koznov $^{8}$, M. V. Malkov ${ }^{8}$,

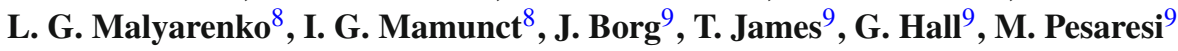 \\ ${ }^{1}$ CERN, European Organization for Nuclear Research, 1211 Geneva 23, Switzerland \\ ${ }^{2}$ Laboratore de l'Accelerateur Lineaire (LAL), Universite Paris Sud Orsay, Orsay, France \\ ${ }^{3}$ INFN Sezione di Roma, Piazzale Aldo Moro 2, 00185 Rome, Italy \\ ${ }^{4}$ INFN Sezione di Napoli, 80126 Naples, Italy \\ ${ }^{5}$ INFN, LNF, Via Fermi, 40, 00044 Frascati, Rome, Italy \\ ${ }^{6}$ NRC Kurchatov Institute-IHEP, 142281 Protvino, Russia \\ 7 Joint Institute for Nuclear Research, Joliot-Curie 6, 141980 Dubna, Russia \\ ${ }^{8}$ Petersburg Nuclear Physics Institute in National Research Centre "Kurchatov Institute", 188300 Gatchina, Russia \\ ${ }^{9}$ Blackett Laboratory, Imperial College, London SW7 2AZ, UK
}

Received: 13 April 2019 / Accepted: 28 November 2019 / Published online: 9 December 2019

(C) The Author(s) 2019

\begin{abstract}
We present the experimental observation of the reduction of multiple scattering of high-energy positively charged particles during channeling in single crystals. According to our measurements the rms angle of multiple scattering in the plane orthogonal to the plane of the channeling is less than half that for non-channeled particles moving in the same crystal. In the experiment we use focusing bent single crystals. Such crystals have a variable thickness in the direction of beam propagation. This allows us to measure rms angles of scattering as a function of thickness for channeled and non-channeled particles. The behaviour with thickness of non-channeled particles is in agreement with expectations whereas the behaviour of channeled particles has unexpected features.
\end{abstract}

\section{Introduction}

It has long been known that the interaction of charged particles with a single crystal differs in many respects from such interactions with an amorphous substance [1-4]. In particular, in single crystals, the effect of planar channeling is observed, when positively charged particles entering the crystal at small angles with respect to the system of crystallographic planes are captured by these planes between adjacent layers of atoms [5]. In the plane orthogonal to the

\footnotetext{
a e-mail: maisheev@ihep.ru
}

crystallographic planes the trajectory of particles is determined by the atomic planar potential. In the direction along the crystallographic planes the particle motion is practically independent of the atomic potential but in principle the particle may interact with individual positively charged atomic nuclei. The result of such interactions is multiple scattering of the particle.

In this paper we investigate the multiple scattering of particles during their channeling within silicon crystals. The investigation is based on measurements of interactions of high energy (180 and $400 \mathrm{GeV} / \mathrm{c}$ ) positively charged particles with focusing bent silicon crystals (with (111) planar orientation) [6]. We compare these results with those for non-channeled particles. We have not seen publications with measurements of multiple scattering when channeling high energy positively charged particles in single crystals. However, in Ref. [7] were presented the results of scattering for non relativistic (3-10 MeV) protons channeled in silicon and germanium single crystals. Besides, for negatively charged particles (electrons with energy in the range 3.35-14 GeV), the electron scattering angle was 1.7 times larger than predicted for an amorphous medium [8].

The problems of the scattering of relativistic particles in straight single crystals were considered in [9-13]. In the paper $[9,10]$ was presented quantum theory of channeled electron and positron scattering in a crystal. Here in particular was predicted that the ordered disposition of the atoms in the crystal plane (chain) leads to the suppression of the 
probability for transition between the states of the transverse motion of a particle in the channel. In Refs. [11,12] is shown that if an ultrarelativistic charged particle intersects crystallographic planes at a sufficiently small angle (but larger than critical angle of channeling), the mean square angle of its deviation in the plane perpendicular to the crystallographic plane decreases in comparison with the case of an amorphous material.

It should be noted that the scattering processes of ultrarelativistic particles in bent crystals should be differ from such processes in straight crystals. Formally, it is easy to understand since in a bent crystal plays an important role of the so-called effective potential [14]. So, due to this, in particular, there is a specific type of scattering, called volume reflection $[15,16]$. It can be expected that between the scattering processes in straight and bent crystals, there is a certain connection, perhaps a similar to connection between the processes of radiation of light leptons or the photoproduction of electron-positron pairs in such crystals and the established in papers $[17,18]$. In addition, the use of bent single crystals allows us to more easily and reliably separate the fraction of channeling particles from non-channeling in comparison with straight crystals.

The paper is organized as follows. First, we give a short description of the experimental setup and describe the procedure of the data analysis. In the subsequent sections we present the experimental results obtained with three crystals in the focusing and defocusing mode, after which a discussion and conclusions follow.

\section{Experimental setup}

The experiment was carried out at the $\mathrm{H} 8$ beam line of the CERN SPS using a practically pure $400 \mathrm{GeV} / \mathrm{c}$ proton beam and a $180 \mathrm{GeV} / \mathrm{c}$ beam of positive secondary particles for the measurements. The layout of the experiment shown in Fig. 1 was similar to that described earlier in [6]. A high precision goniometer was used to orient the crystal planes with the respect to the beam axis with an accuracy of $2 \mu \mathrm{rad}$. The accuracy of the preliminary crystal alignment with a laser beam was about $0.1 \mathrm{mrad}$. Five pairs of silicon microstrip detectors, two upstream and three downstream of the crystal deflector, were used to measure incoming and outgoing angles of particles with an angular resolution in each arm of about $3 \mu \mathrm{rad}[19,20]$. The detector spatial resolution was measured to be about $7 \mu \mathrm{m}$. The geometric parameters of the incident beam were measured with the help of the detector telescope. The width of the beam along the horizontal and vertical axes was a few millimeters. The angular divergence of the incident beam in the horizontal and vertical planes was $\sim 10 \mu \mathrm{rad}$ for the proton beam and $\sim 20 \mu \mathrm{rad}$ (horizontal) and $\sim 40 \mu \mathrm{rad}$ (vertical) for the secondary beam of positively charged particles. The average cycle time of the SPS during the measurements was about $45 \mathrm{~s}$ with a pulse duration of 10-11 s. Typically, the number of particles per spill was $1.310^{6}$.

The experimental configuration described here was used to measure the focusing properties of special single crystals. Such crystals were placed in the goniometer and after the orientation procedure the envelope of the beam was measured (see Fig. 1). Several focusing single crystals were investigated in focusing or defocusing modes. Part of the data obtained in experiments with focusing crystals was used for the present study.

\section{Focusing single crystals}

The first measurements of the beam focusing effect were performed in the 1990s [21]. Since then the focusing devices have been significantly improved [6,22]. Figure 2 illustrates the operation principle of such devices. The focusing crystal is represented by the sum of rectangle $\mathrm{ABCF}$ and triangle FCD (see Fig. 2a). Positively charged particles entering the bent crystal in the channeling regime are deflected through the same angle over the distance BC (AF). For a sufficiently large deflection angle, the channeled and non-channeled particles (background) are spatially separated (see also Fig. 1). The triangular part of the crystal deflects particles with different transverse coordinates $x$ according to a linear relationship between the angle and coordinate. Therefore, the particle trajectories converge at some (focal) point (see Fig. 2b).

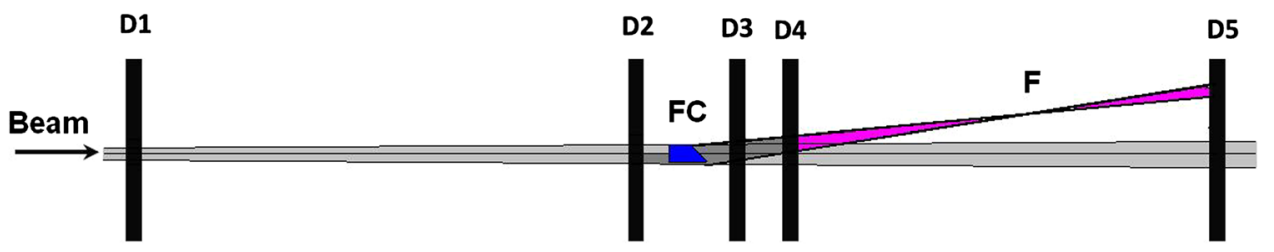

Fig. 1 A schematic view of the layout of the experiment for the measurement of multiple scattering of protons in the crystal deflectors. D1-D5 are the silicon microstrip detectors, FC is a bent focusing silicon crystal. Part of the beam after the bent crystal is deflected and focused at point F (focal point) 
Fig. 2 Focusing crystal: a operation principle; $\mathbf{b}$ the measured two-dimensional plot: deflection angle versus horizontal coordinate $x$; c focusing crystal before installation in the holder

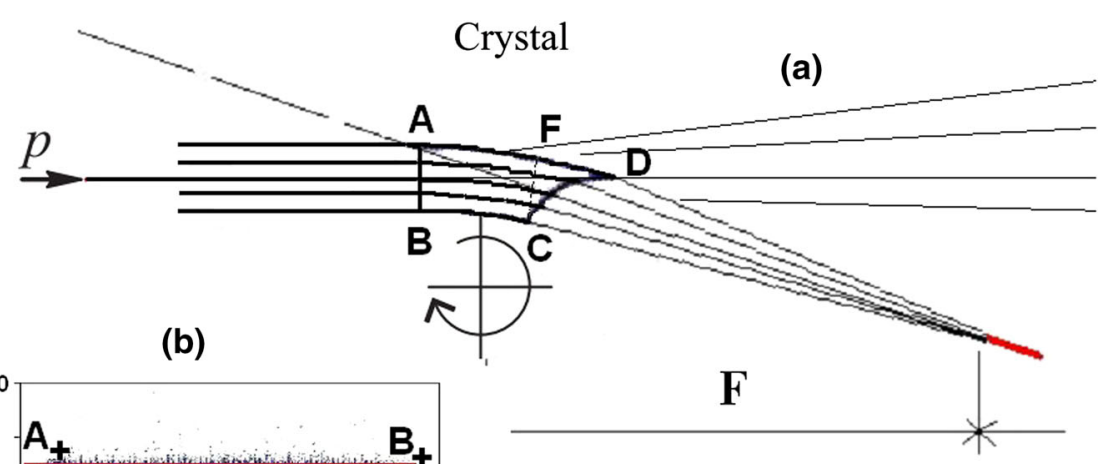

(c)

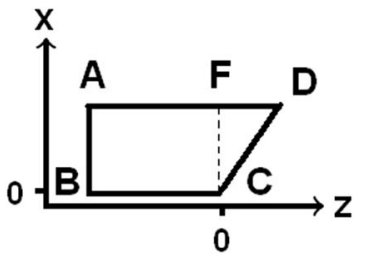

The results of a recent study of strip focusing crystals can be found in [6].

The list of focusing crystals investigated is presented in Table 1 of [6]. For the present study we use the data collected for crystals 1,2 and 4 . Crystal 1 has dimensions: $A B=2.07 \pm 0.01 \mathrm{~mm}, A D=49.84 \pm 0.02 \mathrm{~mm} B C=$ $29.8 \mathrm{~mm}$. Crystal 2 is approximately the same size. Crystal 4 has the same $A D$ and $B C$ sizes as crystal 1 but $A B \approx 4 \mathrm{~mm}$. The height (not shown in the figure) of every crystal was $\approx 86 \mathrm{~mm}$.

\section{Measurements}

The procedure for spatial and angular orientation of the crystal in the goniometer was described in our previous articles [23]. The particle beam passed in the plane (111) of the silicon crystal at an angle of $87 \pm 5 \mathrm{mrad}$ to the crystallographic axis $\langle 110\rangle$. A sufficiently large value of this angle made it possible to completely exclude the influence of the axis on the processes under study. The deflection of a beam occurred in the horizontal plane. The used methods of fabrication and adjustment of focusing crystals allow us to conserve practically identical orientation for all crystals. As a result, for every particle crossing the oriented crystal we obtained: (a) the horizontal and vertical coordinates; (b) the horizontal and vertical incident angles; (c) the horizontal and vertical outgoing angles after the crystal. The difference between the horizontal (vertical) outgoing and incoming angles gives the horizontal (vertical) deflection angle for each particle. Figure $2 b$ illustrates the results in a two-dimensional plot of the horizontal angle of deflection versus the horizontal coordinate. The particles captured in the channeling regime and which passed through the body of crystal in this regime are located between lines $Q_{+} Q_{+}^{\prime}$ and $Q_{-} Q_{-}^{\prime}$. Selected in this way the set of channeled particles undergoes multiple scattering in the vertical direction. Distributions of channeled particles over vertical scattered angles constitute one of the subjects of our study.

For comparison, we also need vertical angular distributions of non-channeled particles. Such particles were selected among particles with an angle of entry into the crystal that exceeds the critical channeling angle. We used data only for particles with an angle of entry directed in the opposite direction to the angle of deflection due to bending.

In addition, the data obtained for each case (channeled and non-channeled particles) were divided into 20 parts for crystals 1 and 2 (40 parts for the crystal 4) according to their horizontal coordinates. So, in section 0 we took particles with horizontal coordinates from -0.05 to $0.05 \mathrm{~mm}$, in section 1 those with horizontal coordinates from 0.05 to $0.15 \mathrm{~mm}$, in section -1 those with horizontal coordinates from -0.05 to $-0.15 \mathrm{~mm}$ and so on. Such a selection of the data allowed us to study the process of multiple scattering for different thicknesses. 


\section{Analysis}

Accordingly to theoretical expectation, planar small angle multiple scattering is described by the Gaussian distribution

$\rho(\theta)=\frac{d N}{d \theta}=\frac{1}{\sqrt{2 \pi} \sigma} \exp -\frac{(\theta-\bar{\theta})^{2}}{2 \sigma^{2}}$,

where $\theta$ is the deflection angle relative to the mean angle $\bar{\theta}$. The value $\sigma$ is the rms of the distribution. We will investigate three different cases of scattering for which we introduce the following definitions: $\sigma_{c}, \overline{\theta_{c}}, \sigma_{y}, \overline{\theta_{y}}$ and $\sigma_{x}, \overline{\theta_{x}}$ are rms and mean values for scattering in the vertical plane during channeling, and in the vertical and horizontal planes far from channeling conditions.

It should be noted that Eq. (1) is valid only for relatively small angles of scattering. At large scattering angles the function $\rho(\theta)$ decreases more slowly than follows from Eq. (1).

We obtain from Eq. (1):

$\ln (\rho(\theta))=\ln \left(\frac{1}{\sqrt{2 \pi} \sigma}\right)-\frac{(\theta-\bar{\theta})^{2}}{2 \sigma^{2}}$

We see from this equation that the function $\ln (\rho(\theta))$ is linear in $(\theta-\bar{\theta})^{2}$. Figure 3 demonstrates the corresponding typical experimental behaviour for one of the $x$-intervals. We see that for not too large values of $(\theta-\bar{\theta})^{2}$ the experimental data coincide with a straight line. The solid lines in this figure were obtained by the method of least squares fitting [24-28]. The range of the fits was up to $\sim 1500 \mu \mathrm{rad}^{2}$ for non-channeled particles and up to $\sim 1000 \mu \mathrm{rad}^{2}$ for channeled particles.

From these approximations we find the values $\sigma_{c}, \sigma_{y}, \sigma_{x}$. Figure 4 shows the experimental distributions of $\rho(\theta)$ functions fitted to Gaussian profiles and the resulting values of $\sigma_{c}, \sigma_{y}, \sigma_{x}$. In a similar way we found the corresponding $\operatorname{rms} \sigma_{c}, \sigma_{y}, \sigma_{x}$ for each interval for crystal 1 , and $\sigma_{c}, \sigma_{y}$ for crystals 2 and 4 . This choice we will discuss below.

The dependence of $\sigma_{c}, \sigma_{y}, \sigma_{x}$ as a function of transverse horizontal $x$ (lower scale) and longitudinal $z$ (upper scale) coordinates are shown in Figs. 5 and 6 . The linear connection between $x$ and $z$-coordinates was

$z[\mathrm{~mm}]=40+x_{0}[\mathrm{~mm}]+k x[\mathrm{~mm}]$

where the coefficient $k$ is equal to 9.7 for crystals 1 and 2 , and 5 for crystal 4 . The variable $x$ was from $\approx-1$ to $1 \mathrm{~mm}$ for crystals 1 and 2 , and from $\approx-2$ to $2 \mathrm{~mm}$ for crystal 4 $\left(x_{0} \approx 0\right)$.

For comparison, we present in the Figs. 5 and 6 the calculated dependence of the multiple scattering angle on the thickness in the amorphous substance. For this we apply recommendation if Ref. [28] to use a Gaussian approximation for the central $98 \%$ of the projected angular distribution, with the rms equal to:

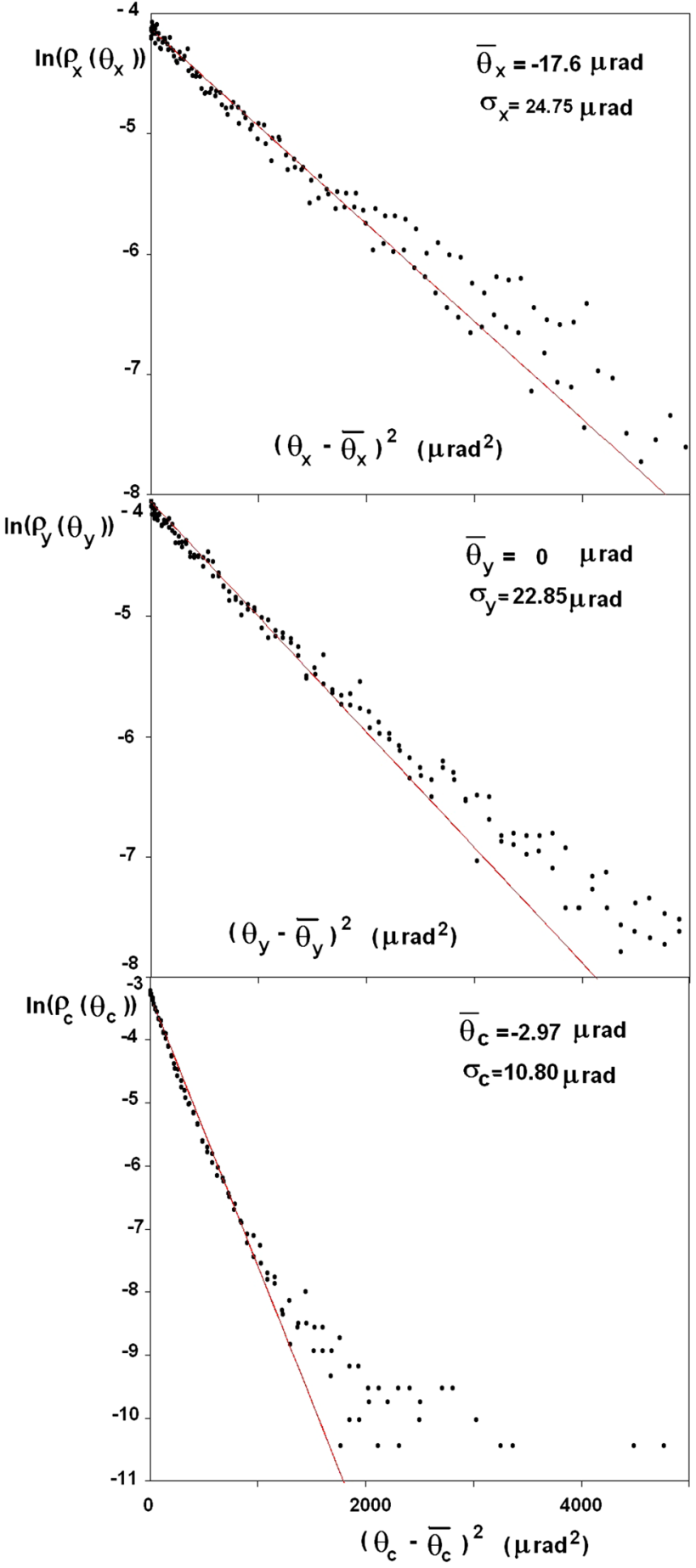

Fig. 3 Experimental angular distributions (for horizontal and vertical non-channeled and vertical channeled particles) plotted on a logarithmic scale in accordance with Eq. (2). The straight lines are fits to linear approximations

$\sigma_{0}=\frac{13.6[M e V]}{\beta c p} \sqrt{l / X_{0}}\left[1+0.038 \ln \left(l / X_{0}\right)\right]$

where $p$ and $\beta c$ are the momentum and velocity of the incident particle, $c$ is the velocity of light, $l, X_{0}$ are the thickness 


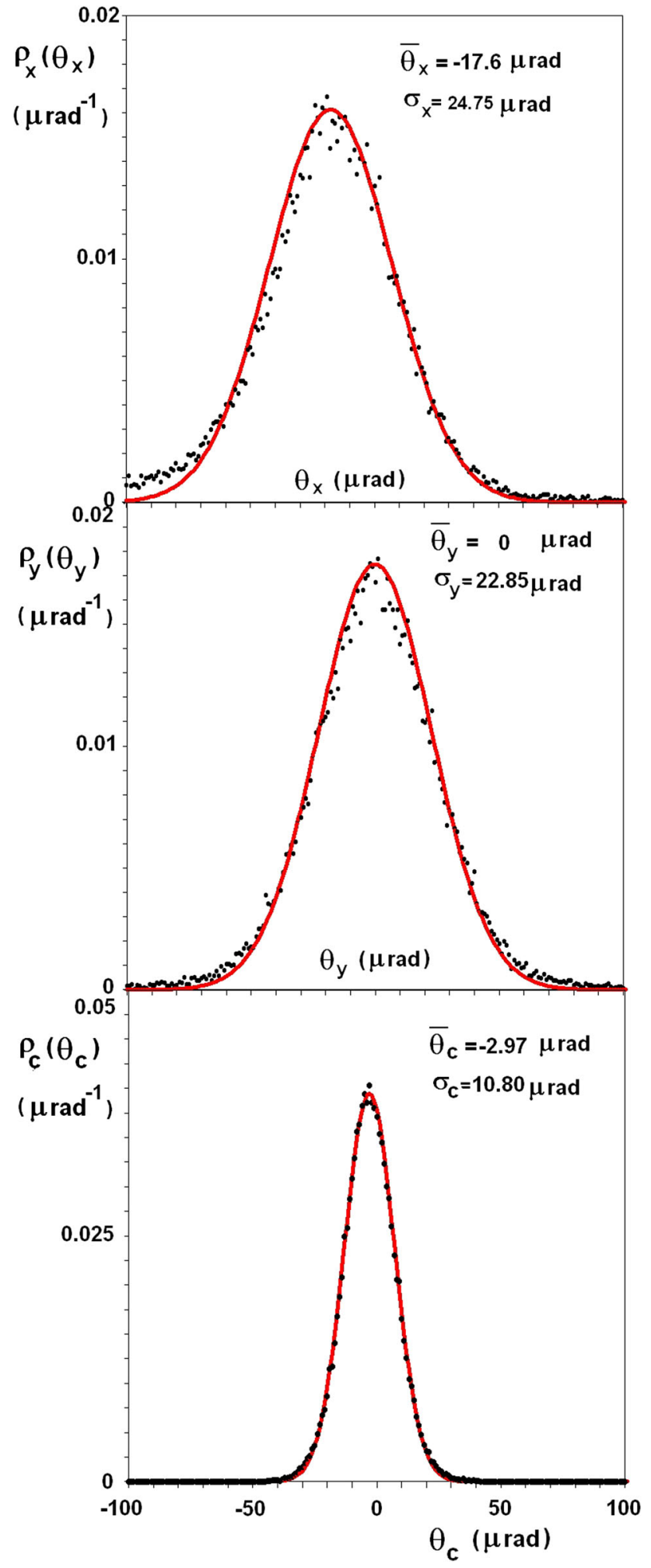

Fig. 4 The particle distributions (from Fig. 3) approximated by Gaussian functions

of the scattering amorphous medium and its radiation length. We use $9.363 \mathrm{~cm}$ for the radiation length of silicon [28].

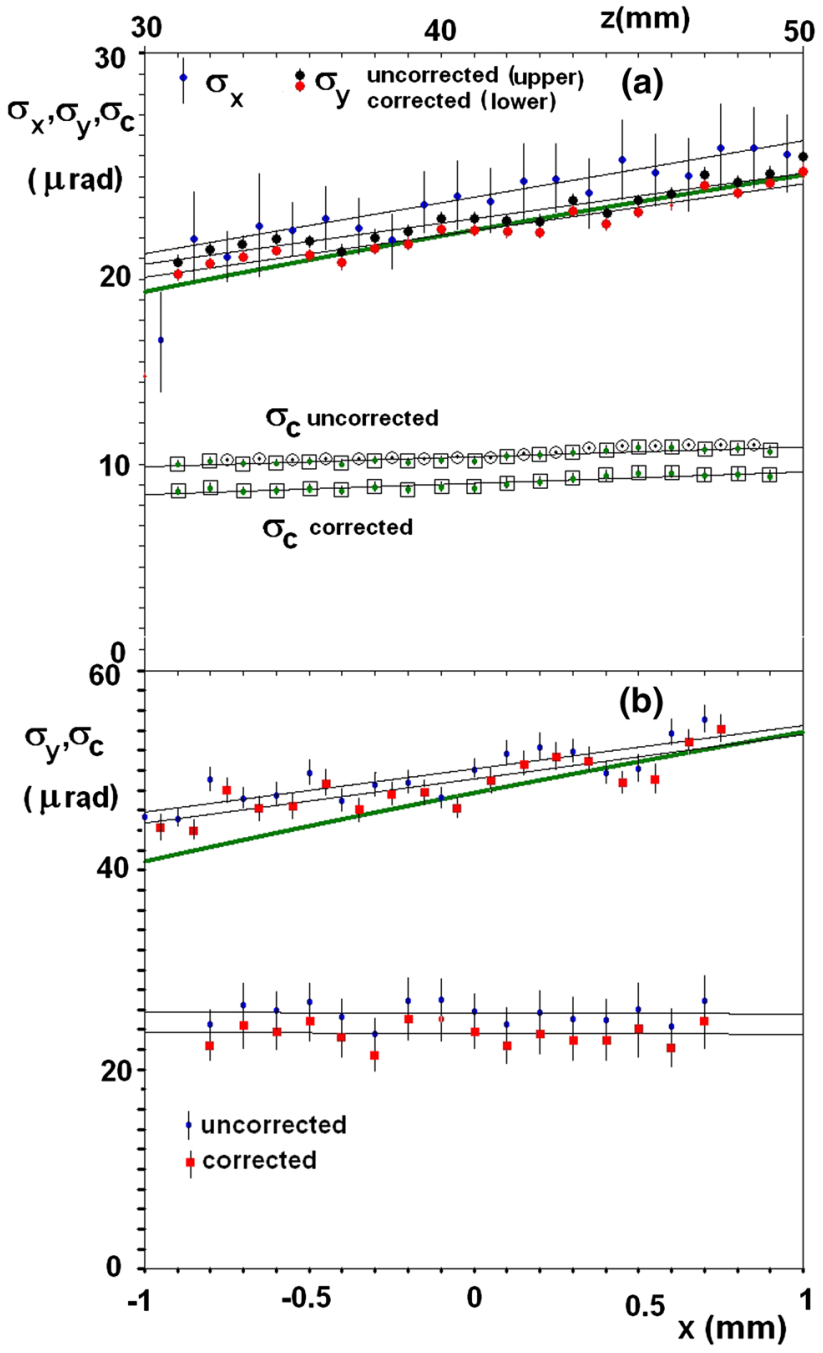

Fig. 5 The $\sigma_{x}, \sigma_{y}$ and $\sigma_{c}$ values as functions of the distance along the crystal (from $30 \mathrm{~mm}$ up to $50 \mathrm{~mm}$ ). The straight lines are linear approximations to the corresponding experimental points. The data are presented for two cases: a beam momentum $400 \mathrm{GeV} / \mathrm{c}$ and crystal 1; b beam momentum $180 \mathrm{GeV} / \mathrm{c}$ and crystal 2 . The lower horizontal scale is the transverse coordinate $x$ and the upper scale is the corresponding longitudinal coordinate $z$. The meaning of corrected and uncorrected results is explained in the text. The small black squares in a represent $\Sigma_{c}$ values (as determined in the text). For better visibility, these squares are offset by $0.05 \mathrm{~mm}$. The green curves are calculations accordingly to Eq. (4). The black thin lines are linear approximations of corresponding experimental results

It should be noted that our collaboration (UA9) has carried out measurements of multiple scattering of $400 \mathrm{GeV} / \mathrm{c}$ protons [29] in single silicon crystals with orientations far from axial or planar channeling. That experiment was performed in parallel and at the same time and on the same installation as the experiments described here with focusing crystals. In [29] the background conditions were investigated. They showed that there is additional scattering of the protons on material in the beam (strip detectors and other matter).Background measurements in the experiment performed without a crystal 


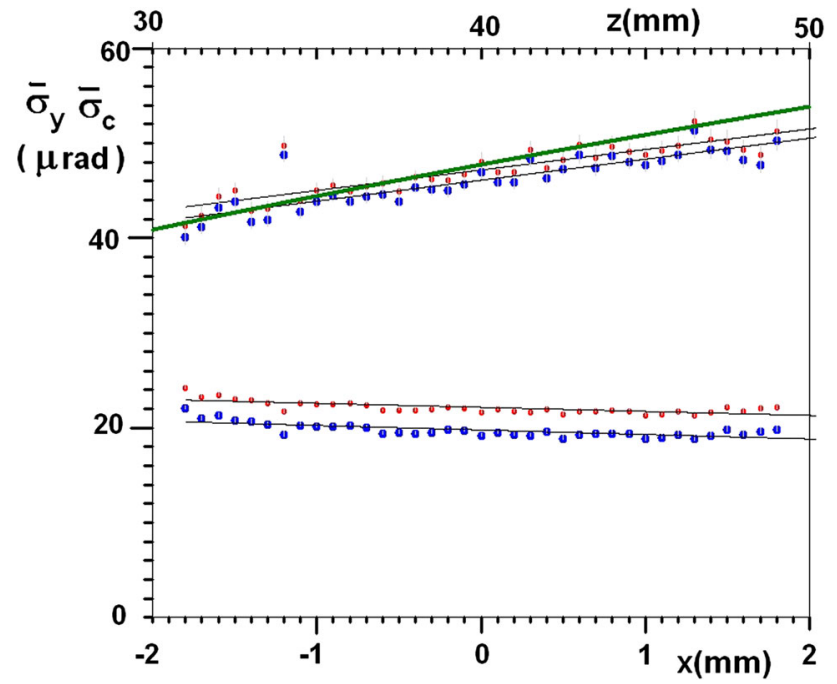

Fig. 6 The $\sigma_{y}$ and $\sigma_{c}$ values as functions of the distance along crystal 4 (from $30 \mathrm{~mm}$ up to $50 \mathrm{~mm}$ ). The beam momentum is $180 \mathrm{GeV} / \mathrm{c}$. The lower horizontal scale is the transverse coordinate $x$ and the upper scale is the corresponding longitudinal coordinate $z$. The red and blue points correspond to uncorrected and corrected (due to background) results. The upper points correspond to $\sigma_{y}$-values and lower points correspond to $\sigma_{c}$-values. The green curves are calculations accordingly to Eq. (4). The black thin lines are linear approximations of corresponding experimental results

show that the contribution of this background process can be described by a Gaussian with rms $\sigma_{b g} \approx 5 \mu \mathrm{rad}$ for a proton beam. Since the rms of multiple scattering angle is inversely proportional to the energy then for the $180 \mathrm{GeV}$ pion beam we chose $\approx 10 \mu \mathrm{rad}$. Then the measured distribution of planar angles $f(\theta)$ can be described by convoluting the background scattering contributions with the scattering distribution in the crystal $f_{s i}$ :

$f(\theta)=f_{b g 1} \otimes f_{s i} \otimes f_{b g 2}$

where $f_{b g 1}$ and $f_{b g 2}$ are the normalized on unit distributions of the background before and after crystal.

Using the properties of convolutions we get

$\sigma_{m}^{2} \approx \sigma_{s i}^{2}+\sigma_{b g}^{2}$

where $\sigma_{m}$ is the rms of measured distribution $f(\theta)$ and $\sigma_{s i}$ is the rms of corrected distribution. This approach is standard and is described, for example, in the recent papers [30,31]. Equation (6) was used in the experiment [29] even under the conditions when $\sigma_{s i} \leq \sigma_{b g}$. In our measurements, the condition $\sigma_{s i} \gg \sigma_{b g}$ was always satisfied, which did not affect the main conclusions of our study.

It is easy to see that the rms multiple scattering (for the same particle energy) differs significantly for channeled and non-channeled particles. For non-channeled particles the rms value is an increasing function of the thickness. For channeled particles this value is a weakly changing function of

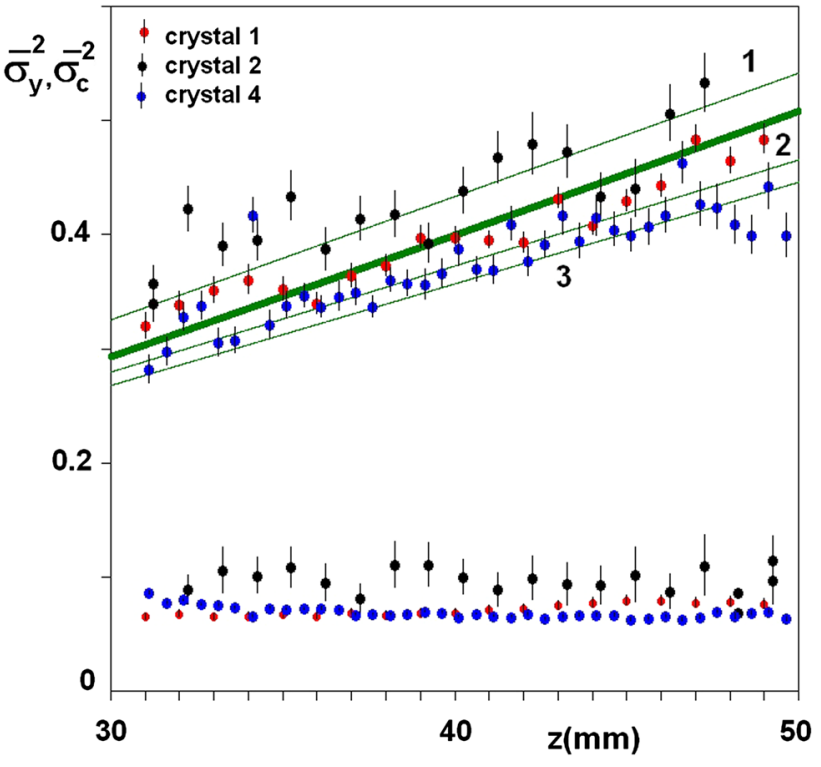

Fig. 7 The mean square values ${\overline{\sigma_{y}}}^{2}=\kappa^{2} \sigma_{y}^{2},{\overline{\sigma_{c}}}^{2}=\kappa^{2} \sigma_{c}^{2}$ (where coefficient $\left.\kappa=E_{0}[\mathrm{MeV}] 10^{6} / 13.6\right)$ as dependences of the crystal thickness. The solid thick curve represents the value $\kappa^{2} \sigma_{0}^{2}$ (see also Eq. (4)). The dependences were obtained for all the crystals at condition with the background corrections. The lines 1, 2 and 3 are drawn with accordance of Eqs. (7) and (8) at boundary values of parameters $A=13.72,12.78,12.42 \mathrm{MeV}$ and $X_{0}^{*}=92,106,112 \mathrm{~mm}$, correspondingly

the thickness. It is interesting that for $400 \mathrm{GeV} / \mathrm{c}$ particles the rms is an increasing function of the thickness while for $180 \mathrm{GeV} / \mathrm{c}$ particles the analogous function is a decreasing function of the thickness. Below we will discuss these results more detail.

In addition, we have the following interesting observation. We computed the value of the function $\Sigma^{2}=\int_{-\theta_{1}}^{\theta_{1}} \theta^{2} \rho(\theta) d \theta$ as a function of $\theta_{1}$. In this relation the function $\rho(\theta)$ is in one case the distribution function over vertical scattered angles of non-channeled particles and in another case the same function for channeled particles; both functions are normalized to unity. We denote the two corresponding $\Sigma^{2}$ functions as $\Sigma_{y}^{2}$ and $\Sigma_{c}^{2}$. As $\theta_{1}$ grows, $\Sigma_{y}^{2}$ increases in value. This is easy to understand due to the non-Gaussian tail of the observed particle distributions. However, for $400 \mathrm{GeV} / \mathrm{c}$ the function $\Sigma_{c}^{2}$ is practically constant and coincident with that calculated by the least square fits.

We see from Eq. (4) that the rms multiple scattering $\sigma_{0}$ of relativistic particles (when $\beta \approx 1$ ) is inversely proportional to their momentum (energy) and the $\sigma_{0}^{2}$ value is proportional to the thickness $l$ (neglecting the logarithmic term). Taking this into account we present in Fig. 7 the results of our measurements of $\sigma_{y}^{2}$ and $\sigma_{c}^{2}$-values divided by a factor $\kappa^{2}$ (where $\left.\kappa=13.6[\mathrm{MeV}] 10^{-6} / E_{0}[\mathrm{MeV}]\right)$ as functions of the $z$ variable. Here $E_{0}=p c$. This shows that all experimental results are consistent with a quadratic dependence on the 
inverse beam energy i.e. $\sigma_{y}^{2}, \sigma_{c}^{2}$ are inverse proportional to $E_{0}^{2}$.

\section{Discussion}

\subsection{Possible explanation of phenomena}

The experimental data demonstrate the reduction of multiple scattering for particles moving in the channeling regime in comparison with such processes for non-channeled particles. This effect has a simple and clear explanation. In amorphous media relativistic particles move at a range of distances (impact parameters) relative to atomic centers (nuclei). This range of impact parameters varies approximately from the radius of the screening of the electric field of the atom to the radius of the atomic nucleus $R_{n} \sim 510^{-13} \mathrm{~cm}$.

A particle captured in the planar channeling regime moves in a space between two adjacent crystallographic planes. At least some of the channeled particles will not be able to get close to the nuclei and hence the scattering angle will be less than in amorphous media. Thus the rms multiple scattering angle depends on the distribution of oscillation amplitudes of channeled particles [32]. From this point of view we can explain the abnormal rms dependence of channeled particles on thickness. Particles with large oscillation amplitudes dechannel during motion and the mean amplitude is decreased.

\subsection{About multiple scattering of non-channeled particles}

Figures 5, 6 and 7 illustrate the behaviour of rms of vertical angle distributions as the functions of thickness for nonchanneled particles. We see that these functions are close enough to the rms for an amorphous medium (see Eq. (2)). Taking into account results of measurements we got approximation description of the experimental data in the follow form

$\sigma_{e}(z)=\frac{13.6[\mathrm{MeV}]}{E_{0}} \sqrt{z / X_{0}^{*}}$,

or in another (equivalent) form

$\sigma_{e}(z)=\frac{A}{E_{0}} \sqrt{z / X_{0}}$

where values $X_{0}^{*}=99 \pm 7 \mathrm{~mm}$ and $A=13.25 \pm 0.47$ $\mathrm{MeV}$ were found from experimental data at $400 \mathrm{GeV} / \mathrm{c}$. Slightly worse conditions were at the momentum of the beam $180 \mathrm{GeV} / \mathrm{C}$ and crystal number 4 . However, we have got $X_{0}^{*}=102 \pm 10 \mathrm{~mm}$ and $A=13.07 \pm 0.65 \mathrm{MeV}$. It should be noted that Eq. (4) was obtained for amorphous media with the accuracy equal to $11 \%$. In this experiment, we studied the multiple scattering of particles in bent silicon single crystals.
Figures 5, 6 and 7 shows a strong dependence of the process on the initial direction of the particle with respect to crystallographic planes. Particles not captured in channeling regime in a bent single crystal are scattered approximately as in the corresponding amorphous medium. However, according to the paper $[11,12]$ in the straight crystal the rms scattering angle should be slightly less by $9 \%$ than in an amorphous medium. However, measurement errors and nonaccuracy of Eq. (4) do not allow us to confirm this prediction.

\subsection{About multiple scattering of channeled particles}

The results presented in Fig. 7 demonstrate reduction of multiple scattering rms during particle channeling by $2-2.3$ times compared to the passage through the crystal of nonchanneled particles. As described above, the value $\Sigma_{c}$ (at a beam momentum of $400 \mathrm{GeV} / \mathrm{c}$ ) is practically independent of $\theta_{1}$ starting from about $45 \mu \mathrm{rad}$ (see also Fig. 5). We ascribe this to reduction of events with large deflection angles due to lack of ability of particles to approach close to nuclei. The comparisons of curves for the crystal 1 with the curves for crystals 2 and 4 demonstrate approximately inverse proportionality with beam momentum for channeled particles. Our explanation of this effect is follows. In the channeling regime some part of particle due to dechanneling process is lost (escape from channeling). One can assume that dechanneling fraction has more large scattering angles. The resulting multiple angle is result of averaging over angles of all the channeling particles at exit from crystal. In other words, mean square angles is result of two competing processes dechanneling and scattering. This can explain the behaviour of the curves in the figures.

On the other hand we see lesser differences between curves for crystals 2 and 4 . These relate to particles of $180 \mathrm{GeV} / \mathrm{c}$ momentum. We can explain this difference because crystal 2 and crystal 4 have different bending radii. It is well-known that the motion of particles in bent crystallographic planes can be described with the help of the effective potential [14]: $U(x)+E_{0} \beta^{2} x / R$, where $x$ is the transverse coordinate and $R$ is the bending radius. The bending radii are $\approx 41 \mathrm{~m}$ and $\approx 200 \mathrm{~m}$ for crystals 2 and 4 , respectively. The corresponding difference of maximum potentials is about $0.3 \mathrm{eV}$.

In the interval $30 \mathrm{~mm}<z<50 \mathrm{~mm}$, the functions $\sigma_{c}$ (see Figs. 5 and 6) were approximated by the linear function const $+A_{0} z$ for each crystal. We got the following values for the slope parameters $A_{0}$ : for the proton beam $0.0499 \pm 0.0028 \mu \mathrm{rad} / \mathrm{mm}$ and $0.0552 \pm 0.0032 \mu \mathrm{rad} / \mathrm{mm}$ for the uncorrected and corrected cases; for the pion beam and crystal 2, respectively, $-0.0081 \pm 0.0278 \mu \mathrm{rad}$ and $-0.0087 \pm 0.0302 \mu \mathrm{rad} / \mathrm{mm}$; and also for the pion beam and crystal $4-0.0852 \pm 0.01464 \mu \mathrm{rad}$ and $-0.09514 \pm$ $0.0169 \mu \mathrm{rad}$. From here it can be seen that for crystal 2 the 
inclination direction (the arithmetic sign of the value $A_{0}$ ) cannot be established due to measurement errors, whereas in the remaining cases the inclination direction is determined reliably enough.

\section{Conclusions}

1. The effect of reduction of multiple scattering of positively charged high-energy particles channeled in single crystals was experimentally observed for the first time with high precision.

2. The multiple scattering behaviour of particles as a function of crystal thickness was experimentally investigated.

3. It was shown that the values of characteristic angles of multiple scattering of channeled and non-channeled particles are inversely proportional to their energy.

4. It was shown that the results of measurements on characteristic angles for multiple scattering of non-channeled particles are consistent with the known formula describing this process, while for channeled particles we see an unexpected effect that the rms scattering angle does not increase significantly for thicknesses from 30 to $50 \mathrm{~mm}$.

5. The effect discovered is also of great practical importance. For example, in $[33,34]$ an application using two focusing crystals for focusing beams in two orthogonal planes was proposed. It can be expected that the effect of reducing multiple scattering will improve the performance of such a system of lenses.

Acknowledgements The IHEP participants of UA9 experiment acknowledge financial support of Russian Science Foundation (grant 17-1201532). The Imperial College group thanks the UK Science and Technology Facilities Council for financial support. The INFN authors acknowledge the support of the ERC Ideas Consolidator Grant No. 615089 CRYSBEAM.

Data Availability Statement This manuscript has no associated data or the data will not be deposited. [Authors' comment: The datasets generated during and/or analysed during the current study available from the corresponding author on reasonable request.]

Open Access This article is licensed under a Creative Commons Attribution 4.0 International License, which permits use, sharing, adaptation, distribution and reproduction in any medium or format, as long as you give appropriate credit to the original author(s) and the source, provide a link to the Creative Commons licence, and indicate if changes were made. The images or other third party material in this article are included in the article's Creative Commons licence, unless indicated otherwise in a credit line to the material. If material is not included in the article's Creative Commons licence and your intended use is not permitted by statutory regulation or exceeds the permitted use, you will need to obtain permission directly from the copyright holder. To view a copy of this licence, visit http://creativecomm ons.org/licenses/by/4.0/.

Funded by SCOAP ${ }^{3}$.

\section{References}

1. M.L. Ter-Mikaelian, High Energy Electromagnetic Processes in Condensed Media (Wiley, New York, 1972)

2. H. Uberall, Phys. Rev. 103, 1055 (1956)

3. H. Uberall, Phys. Rev. 107, 223 (1957)

4. V.N. Baier, V.M. Katkov, V.M. Strakhovenko, Electromagnetic Processes at High Energies in Oriented Single Crystals (World Scientific, Singapore, 1998)

5. J. Lindhard, Influence of crystal lattice on motion of energetic charged particles. Kgl. Dan. Vidensk. Selsk., Mat.-Fys. Medd 34(14), 64 (1965)

6. W. Scandale et al., Phys. Rev. Accel. Beams 21(1), 014702 (2018). https://doi.org/10.1103/PhysRevAccelBeams.21.014702

7. L.C. Feldman, B.R. Appleton, Phys. Rev. B 8, 935 (1973)

8. T.N. Wistisen et al., Phys. Rev. Accel. Beams 19(7), 071001 (2016)

9. V.A. Bazylev, V.V. Goloviznin, Quantum theory of channeled electron and positron scattering in a crystal. Zh. Eksp. Teor. Fiz. 82, 1204 (1982)

10. V.A. Bazylev, V.V. Goloviznin, Quantum theory of channeled electron and positron scattering in a crystal. Sov. Phys. JETP 55, 700 (1982)

11. V.L. Ljuboshits, M.I. Podgoretsky, Multiple Coulomb scattering of ultrarelativistic charged particles moving at small angles to crystallographic planes. Zh. Eksp. Teor. Fiz. 87, 717 (1984)

12. V.L. Ljuboshits, M.I. Podgoretsky, Multiple Coulomb scattering of ultrarelativistic charged particles moving at small angles to crystallographic planes. Sov. Phys. JETP 60, 409 (1984)

13. A.I. Akhiezer, N.F. Shul'ga, Influence of multiple scattering on the radiation of relativistic particles in amorphous and crystalline media. Sov. Phys. Usp. 30, 197-219 (1987)

14. E.N. Tsyganov, Report No. FERMILAB-TM-0682, Batavia (1976)

15. A.M. Taratin, S.A. Vorobiev, Volume reflection of high-energy charged particles in quasi-channeling states in bent crystals. Phys. Lett. A 119, 425 (1987)

16. V.A. Maisheev, Volume reflection of ultrarelativistic particles in single crystals. Phys. Rev. ST Accel. Beams 10, 084701 (2007)

17. V.A. Maisheev, Nuovo Cimento C 34, 73 (2011)

18. S. Bellucci, V.M. Maisheev, Photon emission and electron-positron photoproduction processes in the planar field of a bent single crystal. Phys. Rev. A 86, 042902 (2012)

19. M. Pesaresi, W. Ferguson, J. Fulcher, G. Hall, M. Raymond, M. Ryan, O. Zorba, Design and performance of a high rate, high angular resolution beam telescope used for crystal channeling studies. J. Instrum. 6, P04006 (2011)

20. G. Hall, G. Auzinger, J. Borg, T. James, M. Pesaresi, M. Raymond, A high angular resolution silicon microstrip telescope for crystal channeling studies. Nucl. Instrum. Methods A 924, 175-180 (2019). https://doi.org/10.1016/j.nima.2018.08.060

21. M.A. Gordeeva, M.P. Gurev, A.S. Denisov et al., JETP Lett. 54, 487-490 (1991)

22. W. Scandale et al., Phys. Lett. B 733, 366 (2014). https://doi.org/ 10.1016/j.physletb.2014.05.010 [Corrigendum: Phys. Lett. B 734 (2014) 408. https://doi.org/10.1016/j.physletb.2014.05.051]

23. W. Scandale et al., Phys. Rev. ST Accel. Beams 11, 063501 (2008). https://doi.org/10.1103/PhysRevSTAB.11.063501

24. G.R. Lynch, O.I. Dahl, Nucl. Instrum. Methods Phys. Res. Sect. B 58, 6 (1991)

25. V.L. Highland, Nucl. Instrum. Methods 129, 497 (1975)

26. V.L. Highland, Nucl. Instrum. Methods 161, 171 (1979)

27. K. Nakamura et al., (Particle Data Group), J. Phys. G 37, 075021 (2010)

28. Beringer, et al., (Particle Data Group), Phys. Rev. D 86, 010001 (2012) 
29. W. Scandale et al., Nucl. Instrum. Methods B 402, 291 (2017). https://doi.org/10.1016/j.nimb.2017.02.060

30. N. Berger et al., JINST 9, P07007 (2014)

31. G. Abblende et al., arXiv:1905.11677 (2019)

32. P.N. Chirkov, Y.A. Chesnokov, V.A. Maisheev, D. Bolognini, S. Hasan, M. Prest, E. Vallazza, Int. J. Mod. Phys. A 31(10), 1650051 (2016). https://doi.org/10.1142/S0217751X16500512
33. V.A. Maisheev, Y.A. Chesnokov, New beam optics on the basis of bent single crystals. Nucl. Instrum. Methods B 402, 300 (2017). https://doi.org/10.1016/j.nimb.2017.02.086

34. Y.A. Chesnokov, V.A. Maisheev, High energy neutrino beam generation based on crystal optics. Nucl. Instrum. Methods. A 910, 9 (2018). https://doi.org/10.1016/j.nima.2018.08.116. arXiv:1807.02636 [physics.acc-ph] 